\title{
Long-range propagation of surface plasmons in a thin metallic film deposited on an anisotropic photonic crystal
}

\author{
A. A. Krokhin, A. Neogi, and D. McNeil \\ Department of Physics, University of North Texas, P.O. Box 311427, Denton, Texas 76203, USA
}

(Received 9 April 2007; published 15 June 2007)

\begin{abstract}
We propose using a strongly anisotropic dielectric as a substrate for plasmonic devices. We show that the propagation range of surface plasmons is increased if the substrate is a birefringent dielectric crystal with a properly oriented optical axis. The increase of the propagation range depends on the degree of anisotropy, and, consequently, it turns out to be small for substrates of natural optical crystals, where anisotropy is weak. However, in specially designed photonic crystals, the anisotropy may be very strong, thus leading to appreciable increase of the propagation range. A photonic-crystal substrate, being a medium with nonlinear dispersion, also affects the dispersion law of the surface plasmon. All these effects may increase the efficiency of modern plasmonic and optoelectronic devices.
\end{abstract}

DOI: 10.1103/PhysRevB.75.235420

PACS number(s): 42.70.Qs, 41.20.Jb, 42.25.Lc

\section{INTRODUCTION}

An electromagnetic excitation, propagating along a metaldielectric interface, is known as a surface plasmon (SP). ${ }^{1,2}$ Being a surface mode, it is localized in the vicinity of the interface at distances less than the wavelength, providing a possibility for near-field optical spectroscopy.,4 One more important application of SP is that it acts as a guided carrier in optoelectronic devices. ${ }^{5}$ The resolution of near- and farfield plasmonic devices and the efficiency of optoelectronic devices are essentially determined by the propagation range of the surface plasmon. 3,4,6 The propagation range is limited mostly by the Joule losses, $\mathbf{j} \cdot \mathbf{E}$, in the metallic part of a plasmonic structure. Starting from the pioneering work, ${ }^{7}$ where long-range SP in thin metal film was predicted, much effort has been applied toward the increasing of the propagation range of SP at room temperature. It was shown that the attenuation of one of the SP modes guided along a metal strip imbedded in an isotropic dielectric may be increased by adjusting the width and the thickness of the strip. ${ }^{8}$ Manipulations with the waveguide geometry and the environmental dielectric have led to essential progress in fabrication of quite effective plasmonic waveguides, switchers, and other elements of optoelectronic circuits during the last few years. ${ }^{9}$ For example, an original technique of compensation of Joule losses by cladding a metal film between dielectrics with high optical gain has been recently developed in Ref. 10. An effective method of numerical simulation of the plasmonic waveguides of arbitrary geometry was proposed in Ref. 11.

A plasmonic waveguide has to satisfy two contradictory requirements: long enough propagation range and narrow spatial width. Squeezing in the lateral direction of a SP propagating in a strip with a rectangular cross section leads to higher concentration of electromagnetic energy in the metal and, therefore, to higher level of dissipation. ${ }^{5}$ To reduce the energy in the dissipative medium, it was proposed ${ }^{12}$ to make V-shaped grooves in the metal film, which guide so-called channel surface plasmons. A successful experimental confirmation of this idea opened a lot of possibilities for fabrication of miniature optoelectronic circuits. ${ }^{13}$

In this paper, we propose an alternative method of increasing the propagation range of SP. The scalar product $\mathbf{j} \cdot \mathbf{E}$ can be reduced if the substrate for the metal film is an anisotropic dielectric. In such plasmonic structures, the components of the electric field of SP in the substrate vary with the orientation of the crystallographic axes. For a specific orientation, the scalar product $\mathbf{j} \cdot \mathbf{E}$ can be minimized since the fields in the metal are also effected by the electrodynamic boundary conditions. It is clear that the effect is small if the anisotropy of the substrate is weak. This is the case of natural optical crystal, where anisotropy usually does not exceed a few percents. However, for a substrate that is specially designed to have a large anisotropy, the increase of the propagation range may be quite large. In particular, in twodimensional photonic crystals, the ratio of the ordinary and extraordinary dielectric constants may be as large as $3 .{ }^{14}$ It is known that strong optical anisotropy in artificial dielectric structures may lead to interesting effects, e.g., negative index of refraction in a planar waveguide ${ }^{15}$ or propagation of a surface wave, which does not exist in isotropic medium. ${ }^{16}$

Although the photonic crystals are widely used in optoelectronics, ${ }^{17}$ we are aware of only two examples when a periodic structure (superlattice) was used as a substrate in order (i) to provide an efficient phase matching between the SP and an optical waveguide embedded in the substrate ${ }^{18}$ and (ii) to decrease the Joule losses. ${ }^{19}$

It is clear that, apart from reducing the Joule losses in metal, the photonic-crystal substrates affect the propagation of surface plasmons in many different ways. In particular, it turns out that the penetration depth of the SP increases for the same orientation of the crystallographic axes, providing the longest propagation length. Being a medium with nonlinear dispersion, the photonic-crystal substrate may change the dispersion law of SP. ${ }^{20}$ This occurs if the period of the photonic crystal is comparable with the wavelength of the SP. Due to the periodicity of the substrate along the direction of propagation of SP, the dispersion relation is split into bands. The band structure of SP dispersion relation is well known for periodically corrugated metal surface. ${ }^{3,4}$ In what follows, we consider the propagation of SP in the long-wavelength limit when the plasmon wavelength is much longer than the period of the photonic crystal. In this limiting case, only the lowest band is involved and the substrate can be character- 


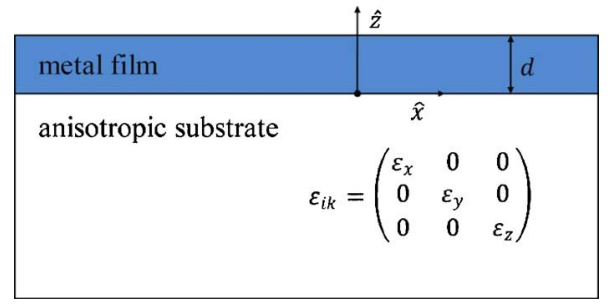

FIG. 1. (Color online) Metal film of thickness $d$ on semi-infinite anisotropic substrate. A surface plasmon propagates along axis $x$. The coordinate axes are directed along the principal axes of the dielectric tensor.

ized by a dispersionless tensor of effective dielectric constants.

\section{DISPERSION AND PROPAGATION RANGE OF SURFACE PLASMON}

We consider an eigenvalue problem for a flat metallic film, which occupies a layer $0<z<d$; the lower surface of the metal layer is in contact with an anisotropic dielectric substrate, $z<0$; the upper semispace, $z>d$, is empty (Fig. 1). The dielectric substrate is an anisotropic optical crystal with principal dielectric constants $\epsilon_{x}, \epsilon_{y}$, and $\epsilon_{z}$.

The dielectric function of the metal is $\epsilon_{m}(\omega)=\epsilon_{m}^{\prime}(\omega)$ $+i \epsilon_{m}^{\prime \prime}(\omega)$. The surface plasmon propagates along the $x$ axis and its magnetic field is parallel to the $y$ axis; $H_{y}$ $=H(x, z) \exp (-i \omega t)$. In the metal, the electric field has longitudinal, $E_{x}=-i\left(c / \omega \epsilon_{m}\right)(\partial H / \partial z)$, and transverse, $E_{z}$ $=i\left(c / \omega \epsilon_{m}\right)(\partial H / \partial x)$, components. In the dielectric, these components are given by $E_{x}=-i\left(c / \omega \epsilon_{x}\right)(\partial H / \partial z)$ and $E_{z}$
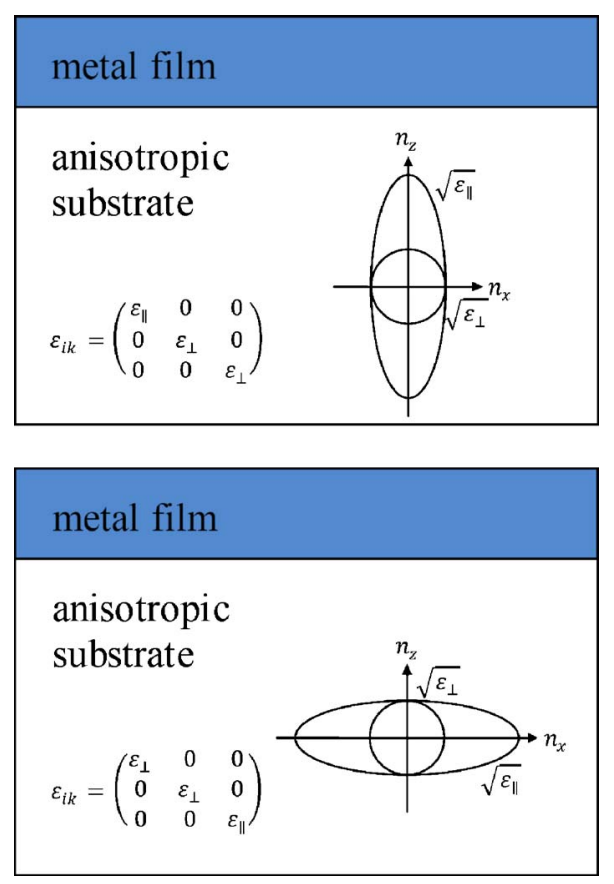

FIG. 2. (Color online) Two orientations, (a) parallel and (b) perpendicular, of the optical axis of the substrate with respect to the metal film. The substrate is a positive uniaxial crystal with $\epsilon_{\|}>\epsilon_{\perp}$.

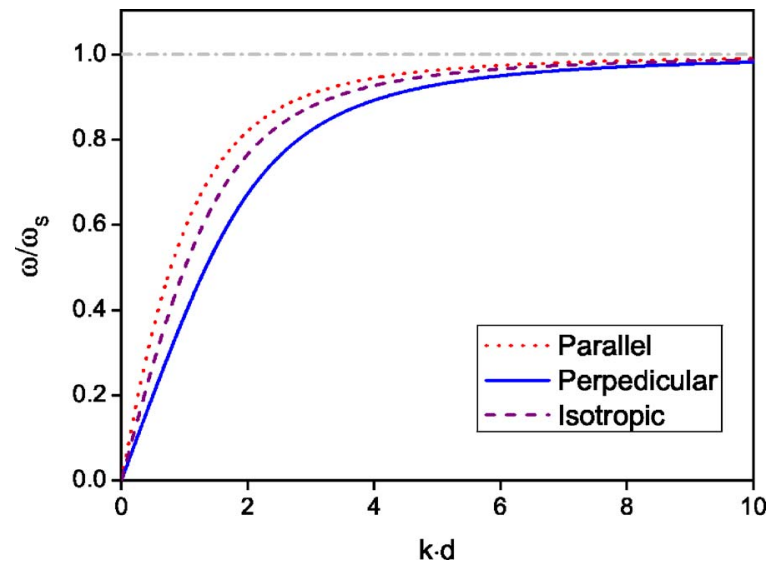

FIG. 3. (Color online) Dispersion curves for SP in a structure with anisotropic substrate. The plasmonic structure is 50-nm-thick silver film on an anisotropic substrate with $\epsilon_{z}=7.5$ and $\epsilon_{x}=2$. The solid (dotted) line is for the orientation of the optical axis perpendicular (parallel) to the metal film. The dashed curve is for the equivalent isotropic substrate with $\epsilon=\sqrt{\epsilon_{x} \epsilon_{z}}=3.87$.

$=i\left(c / \omega \epsilon_{z}\right)(\partial H / \partial x)$. Since the SP is a $p$-polarized wave, the dielectric constant $\epsilon_{y}$ does not appear in the problem. Therefore, in a general case, the substrate can be considered to be a uniaxial crystal with two distinct values of the principal dielectric constants, $\epsilon_{\perp}$ and $\epsilon_{\|}$. They characterize the dielectric properties of the substrate along the optical axis and in the perpendicular plane, respectively. There are two orientations of the optical axis, parallel to the metal film and perpendicular to it. These two geometries are shown in Fig. 2. In what follows, we consider that the substrate is a positive uniaxial crystal, i.e., $\epsilon_{\|}>\epsilon_{\perp}$. In numerical simulations, we use $\epsilon_{\|}=7.5$ and $\epsilon_{\|}=2$. Such strong anisotropy in the longwavelength limit was calculated for photonic crystal of $\mathrm{Si}$ rods arranged in a square lattice with filling fraction $f$ $\approx 0.57 .{ }^{14}$

It follows from the Maxwell equations that inside the dielectric substrate the magnetic field $H(x, z)$ satisfies the Helmholtz equation,

$$
\frac{1}{\epsilon_{z}} \frac{\partial^{2} H}{\partial x^{2}}+\frac{1}{\epsilon_{x}} \frac{\partial^{2} H}{\partial z^{2}}+\frac{\omega^{2}}{c^{2}} H=0 .
$$

The decaying at the $z \rightarrow-\infty$ solution of this equation is $H(x, z)=D \exp \left(i k x+\kappa_{d} z\right)$, where $k$ is the plasmon wave vector and $\kappa_{d}=\sqrt{\epsilon_{x}\left(k^{2} / \epsilon_{z}-\omega^{2} / c^{2}\right)}$ is the inverse penetration depth of the plasmon field inside the substrate. The solution on the vacuum side decays as $z \rightarrow \infty$ and is given by $H(x, z)=A \exp \left(i k x-\kappa_{v} z\right)$, with $\kappa_{v}=\sqrt{k^{2}-\omega^{2} / c^{2}}$. The field inside the metallic film is a superposition of two exponents, $H(x, z)=B \exp \left(i k x+\kappa_{m} z\right)+C \exp \left(i k x-\kappa_{m} z\right), \quad$ with $\kappa_{m}$ $=\sqrt{k^{2}-\epsilon_{m} \omega^{2} / c^{2}}$ being the inverse skin depth. From the continuity of the magnetic field $H(x, z)$ and electric field $E_{x}(x, z)$ at the interfaces $z=0$ and $z=d$, we obtain the dispersion equation for the SP,

$$
\frac{\epsilon_{x} \kappa_{m}+\epsilon_{m} \kappa_{d}}{\epsilon_{x} \kappa_{m}-\epsilon_{m} \kappa_{d}}=e^{-2 \kappa_{m} d} \frac{\kappa_{m}-\epsilon_{m} \kappa_{v}}{\kappa_{m}+\epsilon_{m} \kappa_{v}} .
$$


For the lossless metal with $\epsilon_{m}(\omega) \equiv \epsilon_{m}^{\prime}(\omega)=1-\omega_{p}^{2} / \omega^{2}$ and $\epsilon_{m}^{\prime \prime}(\omega)=0$, this equation gives two real frequencies for any value of $k$. These solutions represent the two branches of the SP. At $k \rightarrow \infty$, the resonant frequencies for these branches are $\omega_{p} / \sqrt{2}$ and $\omega_{s}=\omega_{p} / \sqrt{1+\sqrt{\epsilon_{x}} \epsilon_{z}},{ }^{21}$ where $\omega_{p}$ is the plasma frequency of the metal. The higher-frequency branch originates from the SP, propagating along the metal-vacuum interface. Obviously, it exhibits less sensitivity to the effects of anisotropy than the lower branch, which originates from the SP at the metal-dielectric interface. Therefore, in what follows we study the effects of anisotropy assuming that the dispersion of the SP is determined by the lower branch with the resonant frequency $\omega_{s}=\omega_{p} / \sqrt{1+\sqrt{\epsilon_{\|} \epsilon_{\perp}}}$.

Numerical solutions of Eq. (2) for two orientations of the optical axis are shown in Fig. 3. These curves approach the same resonant frequency at $k \rightarrow \infty$. The third curve between them is the dispersion relation obtained for the structure with equivalent isotropic substrate. Due to the symmetry of the resonant frequency $\omega_{s}$ with respect to the principal dielectric constants $\epsilon_{x}$ and $\epsilon_{y}$, the equivalent isotropic substrate is defined as isotropic dielectric with $\epsilon=\sqrt{\epsilon_{x} \epsilon_{y}}=\sqrt{\epsilon_{\|} \epsilon_{\perp}}$.

In a real metal with finite conductivity $\sigma$, the dissipation does not vanish. The imaginary part of the dielectric function, $\epsilon_{m}^{\prime \prime}=(4 \pi / \omega) \Re \sigma>0$, gives rise to complex values of the wave vector $k=k^{\prime}+i k^{\prime \prime}$ in Eq. (2). Assuming that the dissipation is weak $(\mathfrak{R} \sigma \ll \mathfrak{I} \sigma)$, the propagation range of the SP, $\delta(\omega)=1 / 2 k^{\prime \prime}$, can be calculated perturbatively. In the leading approximation over $1 / \epsilon_{m}^{\prime \prime}$ we obtain from Eq. (2),

$$
\begin{aligned}
\delta(\omega)= & \frac{1}{2 k^{\prime \prime}}=\frac{k}{\epsilon_{m}^{\prime \prime}}\left[\epsilon_{m}\left(\epsilon_{x} \kappa_{v}+\kappa_{d}\right)\left(\epsilon_{m}^{2} \kappa_{v} \kappa_{d}-\epsilon_{x} \kappa_{m}^{2}\right)+d\left(\epsilon_{m}^{2} \kappa_{d}^{2}-\epsilon_{x}^{2} \kappa_{m}^{2}\right)\left(\epsilon_{m}^{2} \kappa_{v}^{2}-\kappa_{m}^{2}\right)-\epsilon_{m} \frac{\kappa_{m}^{2}}{\kappa_{v}}\left(\epsilon_{m}^{2} \kappa_{d}^{2}-\epsilon_{x}^{2} \kappa_{m}^{2}\right)-\epsilon_{m} \frac{\epsilon_{x}^{2}}{\epsilon_{z}} \frac{\kappa_{m}^{2}}{\kappa_{d}}\left(\epsilon_{m}^{2} \kappa_{v}^{2}-\kappa_{m}^{2}\right)\right] \\
& \times\left[\left(\epsilon_{x} \kappa_{v}+\kappa_{d}\right)\left(\epsilon_{m}^{2} \kappa_{v} \kappa_{d}-\epsilon_{x} \kappa_{m}^{2}\right)\left(\kappa_{m}^{2}+k^{2}\right)+\frac{d}{\epsilon_{m}}\left(\kappa_{m}^{2}-k^{2}\right)\left(\epsilon_{m}^{2} \kappa_{d}^{2}-\epsilon_{x}^{2} \kappa_{m}^{2}\right)\left(\epsilon_{m}^{2} \kappa_{v}^{2}-\kappa_{m}^{2}\right)\right]^{-1} .
\end{aligned}
$$

This equation is written in the first nonvanishing approximation over $1 / \epsilon_{m}^{\prime \prime}$; therefore, all the quantities in square brackets in the right-hand side are calculated for a lossless metal, i.e., assuming that $\epsilon_{m}^{\prime \prime}=0$.

\section{ANALYSIS AND NUMERICAL CALCULATIONS}

Equation (3) is valid for a thin metal film when the film thickness is comparable with (or less than) the skin depth, i.e., $d \kappa_{m} \sim 1$. For the case of a thick film, $d \kappa_{m} \gg 1$, the plasmons propagating along the two metal surfaces are decoupled and the dispersion equation (2) is factorized,

$$
\left(\epsilon_{m} \kappa_{d}+\epsilon_{x} \kappa_{m}\right)\left(\epsilon_{m} \kappa_{v}+\kappa_{m}\right)=0 .
$$

In this case, the formula for the propagation range also strongly simplifies. For the SP propagating along the metaldielectric interface, it can be written as follows:

$$
\delta(\omega)=\frac{\epsilon_{m}^{2}-\epsilon_{x} \epsilon_{z}}{\epsilon_{m}^{\prime \prime} \epsilon_{x} k}\left(\frac{\epsilon_{z}-\epsilon_{m}}{\epsilon_{x}-\epsilon_{m}}-\frac{\epsilon_{z}}{\epsilon_{m}}\right)^{-1} .
$$

Since Eqs. (4) and (5) are nonsymmetric with respect to $\epsilon_{x}$ and $\epsilon_{z}$, the propagation range of the SP depends on the orientation of the optical axis of the substrate even in the case of a semi-infinite metal. ${ }^{22}$ The effect of anisotropy is enhanced in a thin metal film, as can be seen from Eq. (3), giving the possibility of controlling the propagation range by orientation of the optical axis of the substrate.

In Fig. 4, we plot the propagation range given by Eq. (3) as a function of the wavelength in vacuum $\lambda=2 \pi c / \omega$. The dispersion relations of Fig. 3 are used for this plot. It is clear that in the infrared region, the perpendicular orientation shown in Fig. 2 is preferential. Here, the propagation range is at least twice as long as the parallel orientation and more than 50\% longer than with an isotropic substrate. However, the difference between the propagation ranges decreases when the frequency approaches the visible range and the effect disappears around $\lambda=0.55 \mu \mathrm{m}$. For shorter wavelengths, the parallel orientation becomes preferential. To understand this nonmonotonic behavior, below we analyze the polarization of the SP.

At low frequencies, the fields of the SP in the dielectric are similar to light fields, i.e., the transversal component $E_{z}$ dominates. Therefore, the slope of the dispersion curves at $\omega \rightarrow 0$ is determined by the $z$ component of the dielectric tensor, i.e., $\omega / c k=1 / \sqrt{\epsilon_{z}}$. The longitudinal component, $E_{x}$, increases with frequency and, close to resonance, the ratio $\left|E_{x} / E_{z}\right|$ reaches its maximum value of $\sqrt{\epsilon_{z} / \epsilon_{x}}$. However, in the metal, the situation is just the opposite. At low frequencies, the dielectric constant of the metal approaches $-\infty$, giving rise to a strong skin effect with the limiting value of the skin depth $\kappa_{m}^{-1} \approx c / \omega_{p} \sim 10^{-5} \mathrm{~cm}$. Under the conditions of skin effect, the transverse component turns out to be much less than the longitudinal component. In fact, $\left|E_{x} / E_{z}\right|$ $=\kappa_{m} / k \rightarrow \infty$ when $k \rightarrow 0$. This means that at low frequencies, the Joule losses are due to the tangential eddy currents. To suppress these currents, it is necessary to suppress the longitudinal component, $E_{x}$, in the metal. This component, being continuous at the metal-dielectric interface, has to be suppressed in the dielectric as well. In the dielectric, $E_{x}=-i\left(c / \omega \epsilon_{x}\right)(\partial H / \partial z)=-i\left(c \kappa_{d} / \epsilon_{x} \omega\right) H$. Since in the longwavelength limit the dispersion of the SP is linear, $\omega$ $=k c / \sqrt{\epsilon_{z}}$, the inverse penetration depth, $\kappa_{d}$ 

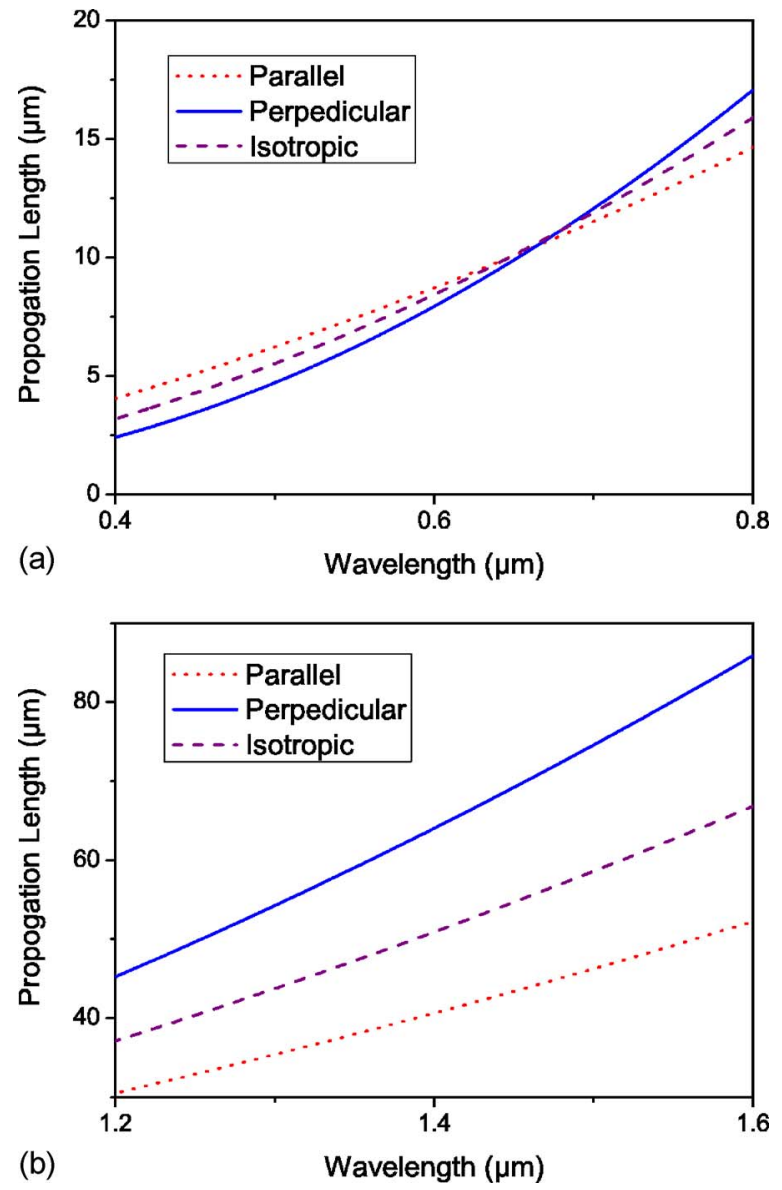

FIG. 4. (Color online) Propagation range of the SP (a) in the optical region and (b) in the infrared region of frequencies.

$=\sqrt{\epsilon_{x}\left(k^{2} / \epsilon_{z}-\omega^{2} / c^{2}\right)}$, vanishes quadratically at $k \rightarrow 0$. The exact expansion at $k \rightarrow 0$ can be obtained from the dispersion equation (2),

$$
\kappa_{d}=k^{2} \frac{\epsilon_{x}}{\epsilon_{z}} \frac{c}{\omega_{p}} \tanh \frac{\omega_{p} d}{c} .
$$

Now, the longitudinal component of the electric field in the dielectric can be written as follows:

$$
E_{x}(x, z)=-i \frac{k c}{\omega_{p} \sqrt{\epsilon_{z}}} \tanh \frac{\omega_{p} d}{c} H(x, z)
$$

It is clear from Eq. (7) that in order to suppress this longitudinal component, it is necessary to increase $\epsilon_{z}$, i.e., the optical density of the substrate in the direction perpendicular to the metal film. The longitudinal component plays a minor role in the dispersion of the SP (it vanishes linearly with $k$ ), but it is just this component that generates dissipative currents at low frequencies. The Joule losses in the metal film, being quadratic over $E_{x}$, are suppressed by the factor $1 / \epsilon_{z}$. In agreement with this estimate, the ratio of the propagation ranges for the two geometries shown in Fig. 2 approaches $\epsilon_{z} / \epsilon_{x}=7.5 / 2 \approx 3.2$ in the long-wavelength limit.

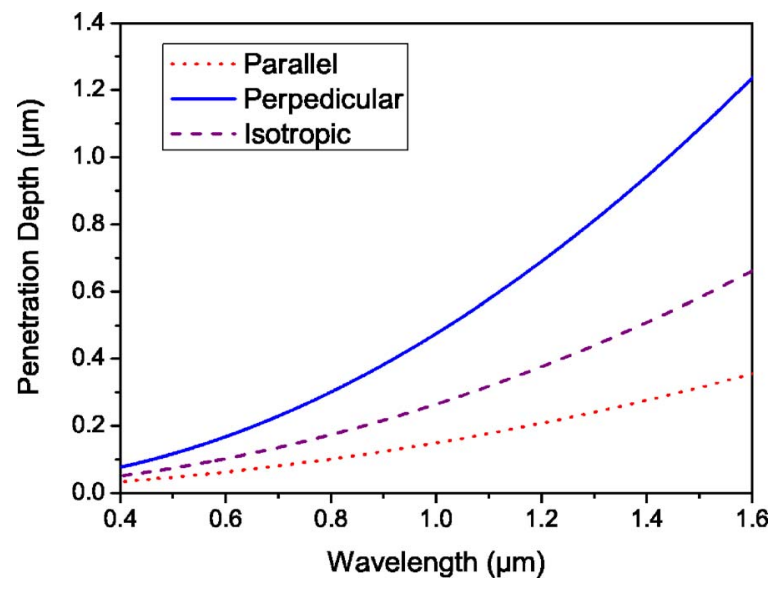

FIG. 5. (Color online) Penetration depth of the electromagnetic field of the SP into the dielectric substrate.

When the frequency increases, the longitudinal component of the SP, $E_{x}$, also increases, and the above considerations become invalid. The role of normal currents in the Joule losses increases with frequency, and eventually the parallel orientation of the optical axis of the substrate becomes preferential. This situation was recently realized in the experiment ${ }^{19}$ where the wavelength of several millimeters was reached. A quantitative comparison of this experiment with our theory is not possible at this stage since the experiment was performed not in the long-wavelength limit and the effects of dispersion play an important role.

Apart from the propagation range, an important characteristic of a plasmonic structure is the penetration depth of the SP field into the dielectric substrate, $\kappa_{d}^{-1}(\omega)$. This parameter determines the strength of coupling of the SP with other elements, e.g., quantum wells, situated below the substrate. It is very desirable from a technological point of view to increase the penetration depth in order to reduce attenuation of the SP fields in the dielectric. Strong decay of the fields in the substrate prevents interaction of the SP with other elements of the integrated photonic circuit usually located below the substrate. In Fig. 5, we plot the penetration depth $\kappa_{d}^{-1}(\omega)$ for two orientations of the optical axis. For the perpendicular orientation, the penetration depth turns out to be longer; thus, this geometry is preferential from this point of view as well. It follows from Fig. 5 that at the telecommunication wavelength $\lambda=1.55 \mu \mathrm{m}$, the penetration depth is increased by a factor of 3 .

\section{CONCLUSIONS}

In conclusion, we demonstrated that the propagation range and the penetration depth of SP can be controlled by the orientation of the optical axis of an anisotropic substrate. In a wide region of frequencies from far to near infrared, the desirable effect is obtained if the optical density of the substrate is the highest in the direction perpendicular to the metal film. For a positive birefringent crystal, it corresponds to the perpendicular orientation of its optical axis with respect to the metal surface. Optical anisotropy of natural crystals is insufficient for appreciable effect; therefore, we pro- 
pose the use of photonic crystals, where optical anisotropies are orders of magnitude stronger than in natural crystals. In particular, a photonic-crystal substrate composed of parallel cylinders may lead to $\approx 100 \%$ increase of the propagation range if the cylinders are oriented perpendicular to the metal film. A layered medium, i.e., one-dimensional (1D) photonic crystal, may be also used as an anisotropic substrate. Since a 1D photonic crystal is a negative birefringent medium, the orientation of the optical axis must then be parallel to the metal surface. This corresponds to orientation of the layers perpendicular to the metal film. At higher frequencies, in the optical and ultraviolet regions, the effect of the anisotropic substrate becomes weaker. It may vanish and even lead to a decrease of the propagation range. The critical frequency when the effect is inverted depends on the thickness of the film and principal dielectric constants of the substrate.

\section{ACKNOWLEDGMENT}

This work is supported by DOE Grant No. DE-FG0206ER46312.
${ }^{1}$ H. Raether, Surface Plasmons (Springer-Verlag, Berlin, 1988).

${ }^{2}$ Surface Polaritons, edited by V. M. Agranovich and D. L. Mills (North Holland, Amsterdam, 1982).

${ }^{3}$ W. L. Barnes, A. Dereux, and T. W. Ebbesen, Nature (London) 424, 824 (2003).

${ }^{4}$ A. V. Zayats, I. I. Smolyaninov, and A. A. Maradudin, Phys. Rep. 408, 131 (2005).

${ }^{5}$ J.-C. Weeber, J. R. Krenn, A. Dereux, B. Lamprecht, Y. Lacroute, and J.-P. Goudonnet, Phys. Rev. B 64, 045411 (2001); B. Lamprecht, J. R. Krenn, G. Schider, H. Ditlbacher, M. Salerno, N. Felidj, A. Leitner, and F. R. Aussenegg, Appl. Phys. Lett. 79, 51 (2001); J.-C. Weeber, M. U. González, A.-L. Baudrion, and A. Dereux, ibid. 87, 221101 (2005); R. Charbonneau, N. Lahoud, G. Mattiussi, and P. Berini, Opt. Express 13, 977 (2005).

${ }^{6}$ H. Ditlbacher, A. Hohenau, D. Wagner, U. Kreibig, M. Rogers, F. Hofer, F. R. Aussenegg, and J. R. Krenn, Phys. Rev. Lett. 95, 257403 (2005); I. I. Smolyaninov, J. Elliott, A. V. Zayats, and C. C. Davis, ibid. 94, 057401 (2005); H. Shin and S. Fan, ibid. 96, 073907 (2006).

${ }^{7}$ D. Sarid, Phys. Rev. Lett. 47, 1927 (1981).

${ }^{8}$ P. Berini, Phys. Rev. B 61, 10484 (2000); 63, 125417 (2001).

${ }^{9}$ T. Nikolajsen, K. Leosson, I. Salakhutdinov, and S. I. Bozhevolnyi, Appl. Phys. Lett. 82, 668 (2003); T. Nikolajsen, K. Leosson, and S. I. Bozhevolnyi, ibid. 85, 5833 (2004); A. Boltasseva, T. Nikolajsen, K. Leosson, K. Kjaer, M. S. Larsen, and S. I. Bozhevolnyi, J. Lightwave Technol. 23, 413 (2005); A. Boltasseva, S. I. Bozhevolnyi, T. Søndergaard, T. Nikolajsen, and K. Leosson, Opt. Express 13, 4237 (2005); P. Berini, R. Charbonneau, N. Lahoud, and G. Mattiussi, J. Appl. Phys. 98,
043109 (2005)

${ }^{10}$ M. A. Noginov, G. Zhu, M. Bahoura, J. Adegoke, C. E. Small, B. A. Ritzo, V. P. Drachev, and V. M. Shalaev, Opt. Lett. 31, 3022 (2006).

${ }^{11}$ A. Degiron and D. R. Smith, Opt. Express 14, 1611 (2006).

${ }^{12}$ J. Q. Lu and A. A. Maradudin, Phys. Rev. B 42, 11159 (1990); I. V. Novikov and A. A. Maradudin, ibid. 66, 035403 (2002).

${ }^{13}$ S. I. Bozhevolnyi, V. S. Volkov, E. Devaux, and T. W. Ebbesen, Phys. Rev. Lett. 95, 046802 (2005); S. I. Bozhevolnyi, V. S. Volkov, E. Devaux, J.-Y. Laluet, and T. W. Ebbesen, Nature (London) 440, 508 (2006).

${ }^{14}$ P. Halevi, A. A. Krokhin, and J. Arriaga, Phys. Rev. Lett. 82, 719 (1999).

${ }^{15}$ V. A. Podolskiy and E. E. Narimanov, Phys. Rev. B 71, 201101(R) (2005).

${ }^{16}$ D. Artigas and L. Torner, Phys. Rev. Lett. 94, 013901 (2005).

${ }^{17}$ Roadmap to Photonic Crystals, edited by S. Noda and T. Baba (Kluwer, Boston, 2003).

${ }^{18}$ M. Skorobogatiy and A. V. Kabashin, Appl. Phys. Lett. 89, 143518 (2006).

${ }^{19}$ V. N. Konopsky and E. V. Alieva, Phys. Rev. Lett. 97, 253904 (2006).

${ }^{20}$ G. Fedorov, S. I. Maslovski, A. V. Dorofeenko, A. P. Vinogradov, I. A. Ryzhikov, and S. A. Tretyakov, Phys. Rev. B 73, 035409 (2006).

${ }^{21}$ B. I. Lembrikov, M. A. Itskovsky, H. Cohen, and T. Maniv, Phys. Rev. B 67, 085401 (2003).

${ }^{22}$ Of course, for an isotropic substrate, $\epsilon_{x}=\epsilon_{z}=\epsilon$, Eq. (5) is reduced to the result of Ref. $1, \delta=\left(\epsilon_{m} / k \epsilon_{m}^{\prime \prime}\right)\left(1+\epsilon_{m} / \epsilon\right)$. 